\title{
eVITAL: A Preliminary Taxonomy and Electronic Toolkit of Health-Related Habits and Lifestyle
}

\author{
Luis Salvador-Carulla, ${ }^{1,2}$ Carolyn Olson Walsh, ${ }^{3}$ Federico Alonso, ${ }^{1,2}$ Rafael Gómez, ${ }^{1}$ \\ Carlos de Teresa, ${ }^{1}$ José Ricardo Cabo-Soler, ${ }^{1}$ Antonio Cano, ${ }^{1}$ and Mencía Ruiz ${ }^{2}$ \\ ${ }^{1}$ Asociación Española para el Estudio Científico del Envejecimiento Saludable (AECES), Calle Infante Don Fernando 17, \\ Málaga, 29200 Antequera, Spain \\ ${ }^{2}$ Asociación Científica PSICOST, Plaza de San Marcos 6, 11403 Jerez, Spain \\ ${ }^{3}$ Harvard Medical School, clo Peabody Society, 260 Longwood Avenue, Boston, MA 02115, USA
}

Correspondence should be addressed to Luis Salvador-Carulla, luis.salvador@telefonica.net

Received 14 October 2011; Accepted 28 November 2011

Academic Editor: Javier Garcia Campayo

Copyright (C) 2012 Luis Salvador-Carulla et al. This is an open access article distributed under the Creative Commons Attribution License, which permits unrestricted use, distribution, and reproduction in any medium, provided the original work is properly cited.

Objectives. To create a preliminary taxonomy and related toolkit of health-related habits $(\mathrm{HrH})$ following a person-centered approach with a focus on primary care. Methods. From 2003-2009, a working group ( $n=6$ physicians) defined the knowledge base, created a framing document, and selected evaluation tools using an iterative process. Multidisciplinary focus groups $(n=29$ health professionals) revised the document and evaluation protocol and participated in a feasibility study and review of the model based on a demonstration study with 11 adult volunteers in Antequera, Spain. Results. The preliminary taxonomy contains 6 domains of $\mathrm{HrH}$ and 1 domain of additional health descriptors, 3 subdomains, 43 dimensions, and 141 subdimensions. The evaluation tool was completed by the 11 volunteers. The eVITAL toolkit contains history and examination items for 4 levels of engagement: self-assessment, basic primary care, extended primary care, and specialty care. There was positive feedback from the volunteers and experts, but concern about the length of the evaluation. Conclusions. We present the first taxonomy of $\mathrm{HrH}$, which may aid the development of the new models of care such as the personal contextual factors of the International Classification of Functioning (ICF) and the positive and negative components of the multilevel person-centered integrative diagnosis model.

\section{Introduction}

Noncommunicable diseases cause 6 out of 10 deaths, and cardiovascular disease alone causes $31.5 \%$ of deaths in female and $26.8 \%$ in males [1]. Many of the leading causes of death have evidence-based modifiable risk factors [2-4], but this does not always translate to healthy behavior by individuals. Several studies have shown that risk of mortality or disease decreases stepwise based on the number of healthy habits practiced by an individual $[5,6]$. In spite of the fact that major chronic diseases are caused by multiple risks, which when combined are associated with health outcomes, the science of multiple health behavior change and assessment is at an early stage, and factors that facilitate or impede success in investigative or clinical intervention in multiple behavior change are unknown [7].

The developing field of longevity medicine takes a holistic view of health that calls for integrative evaluation of health-related habits ( $\mathrm{HrHs})$, both those that increase and decrease risk of disease and those related to general health and well-being, considering the endpoint of years lived without disability and taking into account a person-centered approach [8]. Taxonomies are particularly important in developing fields of study in that they standardize terminology and allow for common understanding of research results; recently proposed examples include the fields of adverse drug reactions [9] and patient-initiated medical errors [10]. In the current study, we present a preliminary taxonomy for 
TABle 1: Metabolic classification based on body mass index and physical activity (eVITAL).

\begin{tabular}{ll}
\hline Body mass index $(\mathrm{BMI})$ & Physical activity \\
\hline (a) Underweight $(\mathrm{BMI}<18.5)$ & (i) Sedentary \\
(b) Normal weight (BMI 18.5-25) & (ii) Daily activity, no \\
& purposeful exercise \\
(c) Overweight (BMI 25-30) & (iii) Regular exercise \\
(d) Obese (BMI $>30)$ & (iv) Very active \\
\hline
\end{tabular}

$\mathrm{HrH}$, as well as the Spanish version of the eVITAL toolkit for clinical evaluation of the lifestyle and related determinants of longevity of an individual.

\section{Methods}

Methods and ethics are described in detail elsewhere [11]. In short, the taxonomy and the related eVITAL toolkit were created using a nominal group technique involving a core group of 6 physicians with expertise in various aspects of longevity medicine and 29 health professionals, including physicians, nurses, and psychologists, in a series of four multidisciplinary focus groups. The model used in the creation of the taxonomy was adapted from the International Classification of Functioning, Disability and Health (ICF) [12] and other documents by the World Health Organization (WHO) [13-15], as well as the multilevel person-centered integrative diagnosis model [16], and the transtheoretical model of stages of change [17] and related model of multibehavior change [18]. According to the ICF a "domain" is "a practical and meaningful set of related physiological functions, anatomical structures, actions, tasks, or areas of life" [12]. "Dimensions" are the identifiable components of every domain. In some cases mutually exclusive domains could not be categorized and subdomains had to be defined (see below).

Entities were organized hierarchically into constructs, domains, subdomains, dimensions, subdimensions, and individual items, and codes were assigned using a hierarchical tree. In this conceptual model, health behaviors are part of $\mathrm{HrH}$, complex behavioral patterns which are closely related to other determinants of health as well as to specific health conditions. $\mathrm{HrH}$ are in turn part of the health lifestyle, which is a key component of the "personal factors" defined in the ICF. These personal factors "are the particular background of an individual's life and living," and these factors comprise, among others, "fitness, lifestyle, habits... overall behaviour pattern and character style, individual psychological assets and other characteristics, all or any of which may play a role in disability at any level" [12].

A demonstration study was performed with 11 adult volunteers who completed the evaluation package followed by an open-ended feedback questionnaire. The assessment package was then revised and computerized, the experts involved in the focus groups evaluated the feasibility of the online toolkit using the criteria of applicability, acceptability, and practicality [54], and responses were used to further refine eVITAL.

\section{Results}

3.1. Domains and Dimensions. The working group and experts revised 7 proposed domains (physical activity, diet, cognition, sleep, stress, psychosocial vitality, and risk behaviors) into the final $6 \mathrm{HrH}$ domains by combining vitality and stress into a single domain combining physical activity and diet into one domain, and dividing "other risk behaviors" into the two domains of substance use and other risk habits (Table 2); the domains of cognition and sleep were unchanged. After discussion regarding the placement of sexuality within the hierarchy, it was decided that, while important for quality of life, sexuality does not meet all of the criteria for domains in terms of contributing to years lived without disability; it was therefore included as a subdimension within the vitality and stress domain. Despite the initial intention to only include evaluation of $\mathrm{HrH}$, the working group decided that the clinical utility of the toolkit would be increased by including an assessment of other determinants and conditions of health specifically related to each basic $\mathrm{HrH}$.

The panel suggested creating an overarching "health lifestyle profile," with 6 subprofiles related to the 6 basic HrHs. A seventh domain, "Health descriptors," includes generic descriptors of health related to longevity, such as social and medical determinants of health and current status of health.

The complete taxonomy developed through this process is shown in Table 3. The preliminary taxonomy includes 6 domains or classes (with diet/exercise further divided into three subdomains: generic, diet, and exercise), 43 dimensions or subclasses, and 141 subdimensions. Once the preliminary taxonomy was defined, codes were assigned to each entity and subentity following a hierarchical tree structure. Letters code the main branches or domains: cognition (c), vitality/stress (v), sleep (s), diet/exercise (de), substance use (s), and other risk habits (r). Each letter is followed by a number for the branches, or dimensions, except for the Prochaska stage of change which is coded within each domain by the letter "s" (see Table 3 ). The complete evaluation schema is shown in Table 4; the toolkit is available online at http://www.longevidad.org/.

Regarding cognition, the working group and expert panels included evaluation tools related to intellectual reserve or to a higher vulnerability to problems with memory or other higher cognitive functions. Tools were selected for the vitality and stress domain to evaluate psychological and social characteristics that are associated with longevity or an improved response to stress and illness. The group decided to include a biologic dimension to this domain due to the evidence linking stress to these components of allostatic load [55]. It was decided that, while diet and exercise have traditionally been considered separate domains, there is sufficient overlap in evaluation, clinical consequences, and intervention strategies that they should be combined. For example, both diet and exercise affect body mass index, which can be combined with activity level to form 16 metabolic types (Table 1). For substance use, the group considered 3 categories: substances that are always harmful 
TABLE 2: eVITAL lifestyle profile related to health habits and the Prochaska stage of change in 11 volunteers.

\begin{tabular}{|c|c|c|c|c|c|c|c|c|}
\hline \multirow{2}{*}{ Subject } & \multirow{2}{*}{ Cognition } & \multirow{2}{*}{$\begin{array}{l}\text { Vitality } \\
\text { and stress }\end{array}$} & \multirow{2}{*}{ Sleep } & \multicolumn{2}{|c|}{ Diet and exercise } & \multirow{2}{*}{$\begin{array}{l}\text { Substance } \\
\text { use }\end{array}$} & \multirow{2}{*}{$\begin{array}{l}\text { Other risk } \\
\text { habits }\end{array}$} & \multirow{2}{*}{ Main health conditions (ICD-10) } \\
\hline & & & & Ex. & Diet & & & \\
\hline 1 & $\uparrow$ & $\uparrow$ & $\downarrow$ & $\downarrow \mathrm{M}$ & $\downarrow \mathrm{P}$ & $\uparrow$ & $\uparrow$ & $\begin{array}{l}\text { Insomnia, sleep apnea, overweight, high } \\
\text { cholesterol }\end{array}$ \\
\hline 2 & $\uparrow$ & $\uparrow$ & - & $-\mathrm{C}$ & $-\mathrm{M}$ & $-\mathrm{M}$ & $\uparrow$ & High cholesterol \\
\hline 3 & $\uparrow$ & $\downarrow$ & $\downarrow$ & $\downarrow \mathrm{P}$ & $\downarrow \mathrm{PR}$ & $\uparrow$ & $\uparrow$ & $\begin{array}{l}\text { Sleep apnea (mild), osteoporosis, } \\
\text { Sjögren's syndrome }\end{array}$ \\
\hline 4 & $\uparrow$ & $\uparrow$ & $\downarrow$ & $\downarrow \mathrm{C}$ & $\downarrow \mathrm{P}$ & $\downarrow \mathrm{C}$ & $\downarrow \mathrm{C}$ & $\begin{array}{l}\text { Sleep apnea, overweight/sedentariness, } \\
\text { nicotine abuse, hypertension }\end{array}$ \\
\hline 5 & $\uparrow$ & $\uparrow$ & $\downarrow$ & $-\mathrm{M}$ & $\downarrow \mathrm{P}$ & $\downarrow \mathrm{P}$ & $\uparrow$ & Sleep apnea (mild), overweight \\
\hline 6 & $\uparrow$ & $\uparrow$ & $\downarrow$ & $-\mathrm{M}$ & $\downarrow \mathrm{M}$ & $\uparrow$ & $\uparrow$ & Sleep apnea (mild) \\
\hline 7 & $\uparrow$ & $\uparrow$ & $\uparrow$ & $-\mathrm{M}$ & $-\mathrm{M}$ & $\uparrow$ & $\uparrow$ & Anxiety \\
\hline 8 & $\uparrow$ & $\downarrow$ & $\downarrow$ & $\downarrow \mathrm{P}$ & $\downarrow \mathrm{P}$ & $\uparrow$ & $\uparrow$ & $\begin{array}{l}\text { Anxiety, insomnia, } \\
\text { overweight/sedentariness, high } \\
\text { cholesterol }\end{array}$ \\
\hline 9 & $\uparrow$ & $\uparrow$ & $\downarrow$ & $\downarrow R$ & $\downarrow \mathrm{R}$ & $\downarrow \mathrm{P}$ & $\uparrow$ & $\begin{array}{l}\text { Sleep apnea (mild), sedentariness, } \\
\text { nicotine abuse, diabetes (type } 2 \text { ) }\end{array}$ \\
\hline 10 & - & $\uparrow$ & $\downarrow$ & $-\mathrm{C}$ & $-\mathrm{P}$ & $\uparrow$ & $-\mathrm{P}$ & Sleep apnea, prostate cancer \\
\hline 11 & - & $\uparrow$ & $\uparrow$ & $-\mathrm{C}$ & $\downarrow \mathrm{C}$ & $\downarrow \mathrm{C}$ & $\downarrow \mathrm{A}$ & Anxiety, overweight, nicotine abuse \\
\hline
\end{tabular}

eVITAL Health stage: good $(\uparrow)$, acceptable $(-)$, needs improvement $(\downarrow)$, the Prochaska stage of change (in domains where this is highly relevant and the global impression rating is acceptable or needs improvement): precontemplation $(\mathrm{P})$, contemplation $(\mathrm{C})$, preparation $(\mathrm{PP})$, action $(\mathrm{A})$, maintenance $(\mathrm{M})$, termination (T), relapse (R). Subjects were 11 adult volunteers in Antequera, Spain, who reported themselves to be healthy. Assessment occurred in 2008.

such as nicotine and cocaine, those that can be health promoting in moderation such as wine and caffeine, and medication abuse, due to the potential harm done by misuse of all types of substances. For each substance, the following factors were considered: type/form, timing of use, amount consumed, degree of abuse, and related psychosocial and medical problems. The expert group separated substance use habits and non-substance-related risk habits due to differences in assessment, intervention, and evidence related to longevity. This final domain, "other risk habits," is divided into treatment nonadherence and other risky behaviors; patient error related to treatment nonadherence is not further delineated within this preliminary taxonomy but has recently been described in detail [10].

3.2. Assessment Package. The evaluation is divided into four levels of increasing complexity, starting with basic self-assessment tools (Level 0) and progressing through assessments that can be completed in a basic primary care visit by a nonphysician provider (Level 1), in an extended primary care visit requiring physician expertise (Level 2), and in specialty care (Level 3 ). Within each level the evaluation is divided into two parts: anamnesis (items related to history) and medical exam.

The anamnesis includes 4 templates, 44 inventories, 22 rating scales, 5 sections (sleep, appetite, fatigue, obsessions, and hypochondriasis) of the semi structured interview "Standardized Polyvalent Psychiatric Interview" (SPPI) (also known by its Spanish acronym EPEP) [40], and 6 subclassification systems (Table 4). We selected assessment instruments that were feasible at each assessment level according to level of complexity and need for trained expertise; when available, we prioritized items that had been standardized in Spain. When instruments were not available, the group designed inventories that should be standardized and validated at a later stage. In all, the full assessment package comprises 1078 items.

The medical exam includes physical exam findings (signs and measures) and laboratory tests. A series of standard indexes have been incorporated. The group designed adjusted indexes of cognitive reserve and body mass index that require future validation (Table 3 ).

The assessment package uses several possible methods of scoring the evaluation. In the simplest, after evaluating each domain, the rater gives a global impression score of the patient's profile for that domain in a 3-point Likert scale (good, acceptable, or needs improvement). These scores can be plotted for each of the domains in a health lifestyle profile and compared to the individual's stage of change for each domain to formulate a plan of care. Figure 1 shows a sample assessment. This type of assessment and its related lifestyle profile can be extended to the dimensions, subdimensions, and types.

3.3. Demonstration Study. Characteristics of the 11 adult volunteers were as follows: mean age 57.45 years (range $43-$ 64), 9 male, marital status: 9 married/1 widow/ 1 single, 6 with university degrees, all upper-middle income. Problems in $\mathrm{HrH}$ were identified in all volunteers: 8 individuals had problems with sleep, 8 with diet, 5 with exercise, 5 with substance use, 2 with other risk habits, and 2 with vitality/stress. Cognitive habits were good or acceptable in 
TABLe 3: Classification system of health-related behaviors (7 domains, 3 subdomains, 43 dimensions, and 141 subdimensions).

\begin{tabular}{|c|c|}
\hline h. Domain: health descriptors & \\
\hline Dimensions (5) & Subdimensions (14) \\
\hline h.1. Sociodemographics & h.1.1. Personal information \\
\hline & h.1.2. Information about partner \\
\hline & h.1.3. Work \\
\hline & h.1.4. Ethnicity and culture \\
\hline h.2. Family history & h.2.1. Ancestors and siblings \\
\hline & H.2.1.1. Longevity \\
\hline & H.2.1.2. Medical history \\
\hline & h.2.2. Descendants \\
\hline & H.2.2.1. Longevity \\
\hline & H.2.2.2. Medical history \\
\hline h.3. Developmental history & h.3.1. Infancy \\
\hline & h.3.2. Childhood \\
\hline & H.3.2.1. Generic \\
\hline & H.3.2.2. Laterality \\
\hline h.4. Past medical history & h.4.1. Diseases/health conditions \\
\hline & H.4.1.1. Generic \\
\hline & H.4.1.2. Related symptoms \\
\hline & h.4.2. Surgical history \\
\hline & h.4.3. History of trauma or injury \\
\hline h.5. Medical treatments & h.5.1. Medications \\
\hline & H.5.1.1. Generic \\
\hline & H.5.1.2. Immunization history \\
\hline & H.5.1.3. Hormonal therapy \\
\hline & h.5.2. Alternative therapies \\
\hline & h.5.3. Other medical care within past year \\
\hline c. Domain: cognition & \\
\hline Dimensions (7) & Subdimensions (19) \\
\hline c.1. Cognitive reserve (CR) & c.1.1. Education level \\
\hline & c.1.2. Current intellectual activity \\
\hline & c.1.3. Current rating \\
\hline & C.1.3.1. Global cognitive reserve (gCR) \\
\hline & C.1.3.2. Global cognitive performance (gCP) \\
\hline & C.1.3.3. Cognitive reserve adjusted for risk (CRar) \\
\hline c.2. Attention & c.2.1. Attention generic \\
\hline c.3. Memory & c.3.1. Verbal semantic memory \\
\hline & C.3.1.1. Unprompted \\
\hline & C.3.1.2. With prompts \\
\hline & c.3.2. Visual memory \\
\hline & C.3.2.1. Items correct \\
\hline & C.3.2.2. Errors \\
\hline c.4. Learning & c.4.1. Learning curve \\
\hline & c.4.2. Consolidation \\
\hline & c.4.3. Primacy \\
\hline & c.4.4. Recency \\
\hline & c.4.5. Errors made during learning \\
\hline & c.4.6. Improvement with repeated exposure \\
\hline
\end{tabular}


TABle 3: Continued.

\begin{tabular}{|c|c|}
\hline c.5. Other intellectual functions & $\begin{array}{l}\text { c.5.1. Visuospatial ability } \\
\text { c.5.2. Motor speed } \\
\text { c.5.3. Perception } \\
\text { c.5.4. Executive function }\end{array}$ \\
\hline c.6. Global cognitive decline & $\begin{array}{l}\text { c.6.1. Global assessment } \\
\text { c.6.2. Spatial/temporal orientation }\end{array}$ \\
\hline c.s - Stage of change & c.s.1. Observed \\
\hline v. Domain: vitality and stress & \\
\hline Dimensions (7) & Subdimensions (28) \\
\hline v.1. Vitality & $\begin{array}{l}\text { v.1.1. Happiness } \\
\text { v.1.2. Optimism } \\
\text { v.1.3. Vital state } \\
\text { v.1.4. Sense of purpose } \\
\text { v.1.5. Sense of control (mastery) } \\
\text { v.1.6. Responsibility } \\
\text { v.1.7. Sexuality }\end{array}$ \\
\hline v.2. Social reserve & $\begin{array}{l}\text { v.2.1. Positive relationships } \\
\text { v.2.2. Isolation } \\
\text { v.2.3. Social support }\end{array}$ \\
\hline v.3. Psychosomatic reserve & $\begin{array}{l}\text { v.3.1. Amiability } \\
\text { v.2.2. Self-restraint } \\
\text { v.3.3. Type behavioral pattern (A, B) } \\
\text { v.3.4. Related health conditions } \\
\text { v.3.4.1. Anxiety } \\
\text { v.3.4.2. Depression } \\
\text { v.3.4.3. Rumination/obsessive tendencies } \\
\text { v.3.4.4. Preoccupation with health }\end{array}$ \\
\hline v.4. Major life events & $\begin{array}{l}\text { v.4.1. Number } \\
\text { v.4.2. Impact }\end{array}$ \\
\hline v.5. Psychological resistance to stress & $\begin{array}{l}\text { v.5.1. Emotional stability } \\
\text { v.5.2. Extraversion } \\
\text { v.5.3. Anxiety (trait) } \\
\text { v.5.4. Distress } \\
\text { v.5.4.1. Internalized distress } \\
\text { v.5.4.2. Psychological distress } \\
\text { v.5.5. Emotional State }\end{array}$ \\
\hline v.6. Basic allostatic load & $\begin{array}{l}\text { v.6.1. Blood pressure } \\
\text { v.6.2. Waist-to-hip ratio } \\
\text { v.6.3. HDL } \\
\text { v.6.4. Total cholesterol/HDL } \\
\text { v.6.5. Glycated hemoglobin } \\
\text { v.6.6. C-reactive protein }\end{array}$ \\
\hline v.s - Stage of change & v.s.1. Observed \\
\hline s. Domain: sleep & \\
\hline Dimensions (5) & Subdimensions (12) \\
\hline s.1. Sleep habits and quality & $\begin{array}{l}\text { s.1.1. Sleep schedule } \\
\text { s.1.2. Sleep quality } \\
\text { s.1.3. Other sleep-related behaviors } \\
\text { s.1.4. Ingestions related to sleep }\end{array}$ \\
\hline
\end{tabular}


TABle 3: Continued.

\begin{tabular}{|c|c|c|}
\hline \multirow[t]{5}{*}{ s.2. Insomnia } & \multicolumn{2}{|l|}{ s.2.1. Generic } \\
\hline & \multicolumn{2}{|l|}{ s.2.2. Pattern } \\
\hline & \multicolumn{2}{|c|}{ S.2.2.1. Onset insomnia } \\
\hline & \multicolumn{2}{|c|}{ S.2.2.2. Middle insomnia } \\
\hline & \multicolumn{2}{|c|}{ S.2.2.3. Late insomnia } \\
\hline \multirow[t]{2}{*}{ s.3. Hypersomnia } & \multicolumn{2}{|c|}{ s.3.1. Somnolence } \\
\hline & \multicolumn{2}{|c|}{ s.3.2. Nonrestorative sleep } \\
\hline \multirow{3}{*}{$\begin{array}{l}\text { s.4. Other sleep problems } \\
\text { (related health conditions) }\end{array}$} & \multicolumn{2}{|c|}{ s.4.1. Sleep apnea } \\
\hline & \multicolumn{2}{|c|}{ s.4.2. Restless legs } \\
\hline & \multicolumn{2}{|c|}{ s.4.3. Other sleep problems } \\
\hline s.s - Stage of change & \multicolumn{2}{|c|}{ s.s.1. Observed } \\
\hline \multicolumn{3}{|l|}{$\begin{array}{l}\text { de. Domain: diet and exercise } \\
\text { de. Subdomains (3) } \\
\text { (i) deg-GENERIC } \\
\text { (ii) Specific } \\
\text { (a) d-Diet } \\
\text { (b) e-Exercise }\end{array}$} \\
\hline Dimensions deg. (3) & \multicolumn{2}{|c|}{ Subdimensions deg. (8) } \\
\hline deg.1. Body composition (BC) & \multicolumn{2}{|c|}{ deg.1.1. BMI (generic) } \\
\hline & \multicolumn{2}{|c|}{ deg.1.2. BMIa (adjusted) } \\
\hline & \multicolumn{2}{|c|}{ deg.1.3. BMIar (adjusted for risk) } \\
\hline \multirow[t]{3}{*}{$\begin{array}{l}\text { deg.2. BC components } \\
\text { (fat and lean body mass) }\end{array}$} & \multicolumn{2}{|c|}{$\begin{array}{l}\text { deg.2.1. Estimated BCC (anthropometrics formulas and standardized } \\
\text { calculation tables) }\end{array}$} \\
\hline & \multicolumn{2}{|c|}{ deg.2.2. Indirect BCC (impedance testing) BC } \\
\hline & \multicolumn{2}{|c|}{ deg.2.3. Direct BCC (DEXA) } \\
\hline \multirow[t]{4}{*}{$\begin{array}{l}\text { deg.3. Metabolic type } \\
\text { (see Tables } 3 \text { and } 4 \text { ) }\end{array}$} & \multirow{4}{*}{\multicolumn{2}{|c|}{$\begin{array}{l}\text { deg.3.1. Classification MT: } 16 \text { types }-5 \text { recommendations levels } \\
\text { deg.3.2. METs } \\
\text { deg.3.2.1. Estimated METs (BMI + daily activity classification). } \\
\text { deg.3.2.2. Indirect METs (interview-oriented food + CPA tables). } \\
\text { deg.3.2.3. Direct METs (nutritional calculator and ergometry). }\end{array}$}} \\
\hline & & \\
\hline & & \\
\hline & & \\
\hline Specific $^{(1)} d+e(4+4=8)$ & d. Diet—subdimension (12) & e. Exercise-subdimension (14) \\
\hline \multirow[t]{6}{*}{ Experiences } & $\begin{array}{l}\text { d.1. Experiences of appetite and } \\
\text { weight change }\end{array}$ & e.1. Fatigue during activity \\
\hline & d.1.1. Change in appetite & e.1.1. Intensity \\
\hline & d.1.2. Change in weight & e.1.2. Frequency \\
\hline & & e.1.3. Fatigue during leisure activities \\
\hline & & e.1.4. Degree of interference with activity \\
\hline & & e.1.5. Duration \\
\hline \multirow[t]{6}{*}{ Habits } & d.2. Diet habits & e.2. Activity habits \\
\hline & d.2.1. Schedule & e.2.1. Self-reported activity level \\
\hline & d.2.2. Perception of diet & e.2.2. Degree of sedentariness \\
\hline & d.2.3. Basic eating habits & e.2.3. Exercise habits \\
\hline & d.2.4. Type of diet & e.2.4. Work-related physical activity \\
\hline & d.2.5. Quality of diet & \\
\hline
\end{tabular}


Table 3: Continued.

\begin{tabular}{|c|c|c|}
\hline \multirow[t]{4}{*}{$\begin{array}{l}\text { Health-related conditions-medical } \\
\text { factors }\end{array}$} & $\begin{array}{l}\text { d.3. Health-related conditions } \\
\text { (medical dietary factors) }\end{array}$ & $\begin{array}{l}\text { e.3. Health-related conditions (medical activity } \\
\text { factors) }\end{array}$ \\
\hline & d.3.1. Digestive problems & e.3.1. Musculoskeletal \\
\hline & d.3.2. Food intolerance & e.3.2. Cardiovascular \\
\hline & d.3.3. Dental problems & e.3.3. Respiratory \\
\hline \multirow[t]{3}{*}{ de.s. Stage of change: habits } & d.s. Stage of change-diet & e.s. Stage of change-exercise \\
\hline & d.s.1. Observed & e.s.1. Observed \\
\hline & d.s.2. Subjective & e.s.2. Subjective \\
\hline \multicolumn{3}{|l|}{ u. Domain: substance use } \\
\hline Dimensions (6) & \multicolumn{2}{|c|}{ Subdimensions (30) } \\
\hline \multirow[t]{5}{*}{ u.1. Medications } & \multicolumn{2}{|l|}{ u.1.1. Type } \\
\hline & \multicolumn{2}{|c|}{ u.1.2. Initiation } \\
\hline & \multicolumn{2}{|c|}{ u.1.3. Intensity of consumption } \\
\hline & \multicolumn{2}{|c|}{ u.1.4. Level of abuse } \\
\hline & \multicolumn{2}{|c|}{ u.1.5. Psychosocial and medical problems } \\
\hline \multirow[t]{5}{*}{ u.2. Caffeine } & \multicolumn{2}{|l|}{ u.2.1. Type } \\
\hline & \multicolumn{2}{|c|}{ u.2.2. Initiation } \\
\hline & \multicolumn{2}{|c|}{ u.2.3. Intensity of consumption } \\
\hline & \multicolumn{2}{|c|}{ u.2.4. Level of abuse } \\
\hline & \multicolumn{2}{|c|}{ u.2.5. Psychosocial and medical problems } \\
\hline \multirow[t]{5}{*}{ u.3. Nicotine } & \multicolumn{2}{|l|}{ u.3.1. Type } \\
\hline & \multicolumn{2}{|c|}{ u.3.2. Initiation } \\
\hline & \multicolumn{2}{|c|}{ u.3.3. Intensity of consumption } \\
\hline & \multicolumn{2}{|c|}{ u.3.4. Level of abuse } \\
\hline & \multicolumn{2}{|c|}{ u.3.5. Psychosocial and medical problems } \\
\hline \multirow[t]{5}{*}{ u.4. Alcohol } & u.4.1. Type & \\
\hline & u.4.2. Initia & \\
\hline & u.4.3. Inten & sumption \\
\hline & u.4.4. Level & \\
\hline & u.4.5. Psych & d medical problems \\
\hline u.5. Illicit Drugs & u.5.1. Type & \\
\hline & u.5.2. Initia & \\
\hline & u.5.3. Inten & sumption \\
\hline & u.5.4. Level & \\
\hline & u.5.5. Psych & d medical problems \\
\hline u.s. Stage Of Change & u.s.1. Medic & \\
\hline & u.s.1.1. C & \\
\hline & u.s.1.2. S & \\
\hline & u.s.2. Caffei & \\
\hline & u.s.2.1. O & \\
\hline & u.s.2.2.S & \\
\hline & u.s.3. Nicoti & \\
\hline & u.s.3.1. & \\
\hline & u.s.3.2. $\mathrm{s}$ & \\
\hline & u.s.4. Alcoh & \\
\hline & u.s.4.1. C & \\
\hline & u.s.4.2. S & \\
\hline & u.s.5. Illicit & \\
\hline & u.s.5.2. C & \\
\hline & u.s.5.1.S & \\
\hline
\end{tabular}


TABLE 3: Continued.

\begin{tabular}{ll}
\hline r. Domain: other health risk habits & Subdimensions (4) \\
\hline Dimensions (2) & r.1.1. Generic \\
r.1. Nonadherence to treatment & r.1.s.1. Observed \\
& r.1.s.2. Subjective \\
\hline r.2. Other risk behaviors & r.2.1. Type \\
r.2.2.1. Risky sexual behavior \\
r.2.2.2. Dangerous sports \\
r.2.2.3. Gambling \\
r.2.2.4. Dangerous driving \\
r.2.2.5. Other risk behaviors (e.g., sun exposure) \\
r.2.s Stage of change \\
r.2.s.1. Observed \\
r.2.s.2. Subjective \\
\hline
\end{tabular}

${ }^{(1)}$ Specific subdimensions of diet/exercise are listed one level down from where they are in other domains.

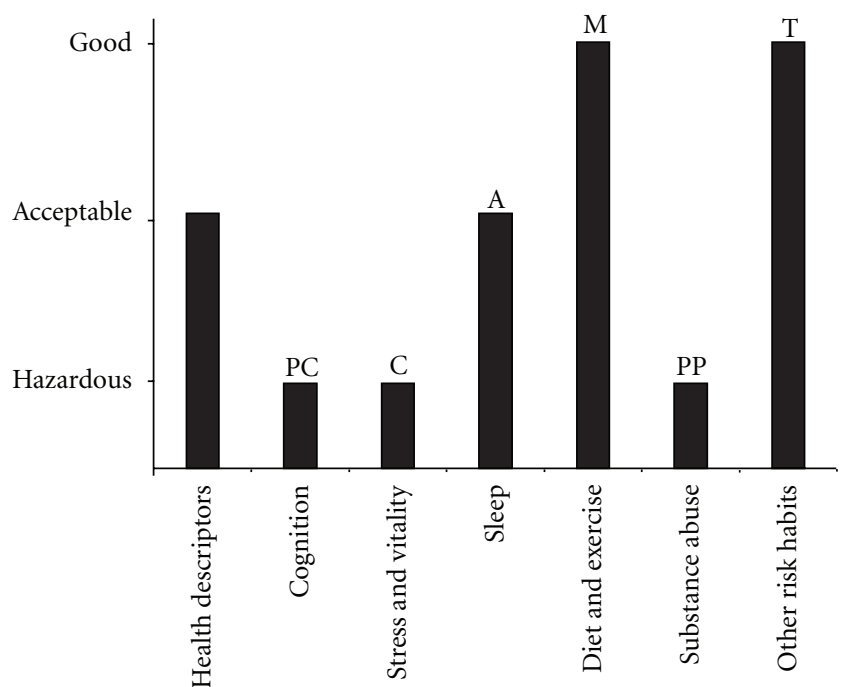

Figure 1: Sample lifestyle profile based on eVITAL toolkit. Prochaska stages: precontemplation $(\mathrm{P})$, contemplation $(\mathrm{C})$, preparation $(\mathrm{PP})$, action $(\mathrm{A})$, maintenance $(\mathrm{M})$, termination $(\mathrm{T})$.

all individuals. 10 individuals fulfilled at least one diagnosis from the International Classification of Diseases (ICD-10) [56] in spite of perceiving themselves as "healthy" (Table 2).

After completing the toolkit, 9/11 gave an overall favorable review and 11/11 reported favorable interactions with the professionals administering the evaluation. While there were no specific recommendations for changes from the volunteers, 7/11 reported that the evaluation was quite long.

3.4. Feasibility Study. Upon reviewing the results of the demonstration study, the working group and focus groups revised the basic organization of the assessment package. Then a feasibility questionnaire was sent to the 29 experts involved in the focus groups; 15 responses were received suggesting changes while 14 experts judged the previous package as adequate and provided no further comments. Comments about applicability of the survey were generally positive. In terms of acceptability, there was some concern about generalizability to populations with lower education level and socioeconomic status, as well as whether patients would be able to complete the forms without assistance. Regarding practicality, there was concern about the time required of the clinician, as well as the difficulty of managing all of the data gathered. As the ultimate goal is to integrate eVITAL into use in the primary care system, comments from primary care practitioners, such as the following, were particularly important: "The survey seems too ambitious and impractical for primary care... a tool that you cannot use due to lack of resources (above all, time) loses its practical validity."

3.5. Development of the Toolkit. These comments were taken into account in developing the electronic toolkit eVITAL. The open access preliminary version of the toolkit is available at http://www.longevidad.org/inicio.

\section{Discussion}

Although there is an increasing interest in the comprehensive assessment of $\mathrm{HrHs}$ and their relationship to longevity [57], this study presents the first attempt at classifying $\mathrm{HrHs}$ to date using the longevity model with the endpoint of years lived without disability. The ICF indicates the relevance of $\mathrm{HrH}$ and lifestyle as main components of the "personal contextual factors," but these factors have not been defined or coded to date [58].

The transtheoretical model of stages of change [17] with the related multibehavioral assessment [18] is the main integrative approach to $\mathrm{HrH}$. Despite the limited evidence regarding the effectiveness of stage-based interventions as a basis for behavior change or for facilitating stage progression 
TABLE 4: Evaluation of items included in the eVITAL toolkit ${ }^{(1)}$.

\begin{tabular}{|c|c|}
\hline \multicolumn{2}{|c|}{ 1. Domain: health descriptors } \\
\hline Evaluation & Instruments \\
\hline History-Level 0 & Sociodemographic inventory \\
\hline \multirow[t]{4}{*}{ History—Level 1} & Family medical history \\
\hline & Personal medical history \\
\hline & Medication, hormone use, and alternative treatments \\
\hline & Immunization history \\
\hline \multicolumn{2}{|l|}{ History-Level 2} \\
\hline \multicolumn{2}{|l|}{ History-Level 3} \\
\hline \multicolumn{2}{|l|}{ Examination-Level 0} \\
\hline \multicolumn{2}{|l|}{ Examination-Level 1} \\
\hline \multicolumn{2}{|l|}{ Examination-Level 2} \\
\hline \multicolumn{2}{|l|}{ Examination-Level 2} \\
\hline \multicolumn{2}{|l|}{ 2. Domain: cognition } \\
\hline Evaluation & Instruments \\
\hline History-Level 0 & Screening for cognitive problems \\
\hline History-Level 1 & Cognitive reserve $(\mathrm{CR})$ \\
\hline \multirow[t]{3}{*}{ History-Level 2} & (i) Cognitive risk factors \\
\hline & (ii) Cognitive reserve adjusted for cognitive risk (CRar) \\
\hline & (iii) Stage of change, objective [17] \\
\hline History-Level 3 & Global cognitive reserve (gCR) \\
\hline \multicolumn{2}{|l|}{ Examination-Level 0} \\
\hline \multicolumn{2}{|l|}{ Examination-Level 1} \\
\hline Examination-Level 2 & 7 -minute test $[19,20]$ \\
\hline \multirow[t]{4}{*}{ Examination-Level 3} & (i) Luria’s test [21] \\
\hline & (ii) Benton's visual recognition test [22] \\
\hline & (iii) Trail making test-A (TMT-A) [23] \\
\hline & (iv) Finger electronic tapping test (FETT) [24] \\
\hline \multicolumn{2}{|c|}{ 3. Domain: vitalty and stress } \\
\hline Evaluation & Instruments \\
\hline History-Level 0 & Screening for emotional state \\
\hline \multirow[t]{5}{*}{ History-Level 1} & (i) Optimism-LOT-R: life orientation test-revised [25] \\
\hline & (ii) Emotional stress-Questions 5-9, SF-36: social functioning-36 question scale [26, 27]/MHI-5. \\
\hline & (iii) Social isolation-STAKES [28] \\
\hline & (iv) Social support—Oslo [29] \\
\hline & (v) Anxiety/depression-HAD: hospital anxiety and depression scale [30] \\
\hline \multirow[t]{5}{*}{ History-Level 2} & (i) NEO Pi-R: revised NEO personality inventory [31] \\
\hline & (ii) Psychological well-being $[32,33]$ \\
\hline & (iii) Sexuality quality of life: sexuality subscales $[34,35]$ \\
\hline & (iv) Stress-HAD $[30,36]$ \\
\hline & (v) Stage of change, objective [17] \\
\hline \multirow[t]{6}{*}{ History-Level 3} & (i) Social readjustment rating scale [37] \\
\hline & $\begin{array}{l}\text { (ii) Type A personality—ERCTA: escala retiro de patrón de conducta tipo A (scale of Type A behavior } \\
\text { pattern) [38] }\end{array}$ \\
\hline & (iii) Aggression subscale_ZKPQ: Zuckerman-Kuhlman’s personality questionnaire [39] \\
\hline & (iv) Obsessions_-SPPI: standardized polyvalent psychiatric interview [40] \\
\hline & (v) Hypochondriasis—SPPI [40] \\
\hline & (vi) Gender-specific medical evaluation, including breast cancer risk assessment [41] \\
\hline
\end{tabular}


Table 4: Continued.

\begin{tabular}{|c|c|c|}
\hline \multicolumn{3}{|l|}{ Examination-Level 0} \\
\hline Examination-Level 1 & & \\
\hline Examination-Level 2 & \multicolumn{2}{|c|}{$\begin{array}{l}\text { Basic allostatic load: blood pressure, waist-to-hip ratio, total/HDL cholesterol, HDL, glycated } \\
\text { hemoglobin, CRP }\end{array}$} \\
\hline Examination-Level 3 & \multicolumn{2}{|c|}{ Expanded allostatic load: add DHEA-S and urinary cortisol } \\
\hline \multicolumn{3}{|l|}{ 4. Domain: sleep } \\
\hline Evaluation & \multicolumn{2}{|l|}{ Instruments } \\
\hline History-Level 0 & \multicolumn{2}{|c|}{ Screening for insomnia and hypersomnia } \\
\hline \multirow[t]{4}{*}{ History-Level 1} & \multicolumn{2}{|c|}{ (i) Insomnia-SPPI [40] } \\
\hline & \multicolumn{2}{|c|}{ (ii) Somnolence-Epworth sleepiness scale [42-44] } \\
\hline & \multicolumn{2}{|c|}{ (iii) Specific sleep-related symptoms } \\
\hline & \multicolumn{2}{|c|}{ (iv) Sleep habits } \\
\hline History-Level 2 & \multicolumn{2}{|c|}{ Stage of change, objective [17] } \\
\hline \multicolumn{3}{|l|}{ History-Level 3} \\
\hline \multicolumn{3}{|l|}{ Examination-Level 0} \\
\hline \multicolumn{3}{|l|}{ Examination-Level 1} \\
\hline \multicolumn{3}{|l|}{ Examination-Level 2} \\
\hline Examination-Level 3 & \multicolumn{2}{|l|}{ Polysomnography } \\
\hline \multicolumn{3}{|c|}{ 5. Domain: diet and exercise } \\
\hline \multirow{2}{*}{ Evaluation } & & Instruments \\
\hline & Instruments DIET & Instruments EXERCISE \\
\hline History-Level 0 & $\begin{array}{l}\text { Screening for change in appetite } \\
\text { (SSPI) }\end{array}$ & Activity level \\
\hline \multirow[t]{7}{*}{ History-Level 1} & Eating habits & Exercise habits \\
\hline & Table of mealtimes & $\begin{array}{l}\text { Exercise readiness_-Par-Q: physical activity } \\
\text { readiness questionnaire [45] }\end{array}$ \\
\hline & Appetite-SPPI [40] & Fatigue-adapted SPPI [40] \\
\hline & Subclassification BMI & Estimated METs \\
\hline & $\begin{array}{l}\text { Metabolic classification (combir } \\
\text { levels) }\end{array}$ & es weight status and activity level) (16 \\
\hline & Classification as recommendatic & ns: 5 levels \\
\hline & $\begin{array}{l}\text { Readiness to change-diet and } \\
\text { exercise-subjective }\end{array}$ & $\begin{array}{l}\text { Readiness to change-diet and } \\
\text { exercise-objective }\end{array}$ \\
\hline \multirow[t]{3}{*}{ History-Level 2} & $\begin{array}{l}\text { CFCA-food frequency } \\
\text { questionnaire }[46]\end{array}$ & \\
\hline & $\begin{array}{l}\text { Digestive symptoms, food } \\
\text { intolerance, dental problems }\end{array}$ & Musculoskeletal physical barriers to exercise \\
\hline & $\begin{array}{l}\text { Readiness to change-diet and } \\
\text { exercise-objective }\end{array}$ & $\begin{array}{l}\text { Readiness to change-diet and } \\
\text { exercise-objective }\end{array}$ \\
\hline \multicolumn{3}{|l|}{ History-Level 3} \\
\hline \multirow[t]{2}{*}{ Examination-Level 0} & \multicolumn{2}{|l|}{ (i) Waist circumference } \\
\hline & \multicolumn{2}{|c|}{ (ii) Reported body mass index (BMI) from reported weight and height } \\
\hline \multirow[t]{3}{*}{ Examination-Level 1} & \multicolumn{2}{|c|}{ (i) Body composition—subclassification BMI } \\
\hline & (ii) Waist-to-hip ratio & \\
\hline & (iii) BMI adjusted for waist circl & mference [47], (BMIa) \\
\hline Examination-Level 2 & $\begin{array}{l}\text { (i) Basic lab work: total/HDL ch } \\
\text { C-reactive protein, microalbum }\end{array}$ & $\begin{array}{l}\text { lesterol, HDL, glycated hemoglobin, } \\
\text { n, glucose, insulin }\end{array}$ \\
\hline & (ii) Blood pressure, heart rate. & \\
\hline & $\begin{array}{l}\text { (iii) Adjusted BMI (for adult we } \\
\text { presence or absence of sleep apn }\end{array}$ & $\begin{array}{l}\text { ght gain, triglyceride/HDL, blood pressure, fasting glucose, and } \\
\text { ea and osteoarthritis (BMIar)) }\end{array}$ \\
\hline & (iv) Body composition (triceps s & kin fold), estimated muscle mass (arm circumference) \\
\hline & (v) Insulin resistance-HOMA-IF & {$[48]$} \\
\hline
\end{tabular}


TABle 4: Continued.

\begin{tabular}{lll}
\hline Examination-Level 3 & $\begin{array}{l}\text { 3-day food record analyzed by } \\
\text { nutritionist }\end{array}$ & $\begin{array}{l}\text { Indirect METs calculated through compendium } \\
\text { of physical activities_CPHA [49] }\end{array}$ \\
& $\begin{array}{ll}\text { Hand strength via dynamometry } \\
\text { (i) Body composition via BIA or }\end{array}$ & $\begin{array}{l}\text { (i) Somatometry } \\
\text { DEXA }\end{array}$ \\
& $\begin{array}{ll}\text { (ii) Nutritional calculator } & \text { (ii) Ergometry } \\
& \text { (iii) Direct METs measurement }\end{array}$ \\
\hline
\end{tabular}

\begin{tabular}{|c|c|}
\hline \multicolumn{2}{|c|}{ 6. Domain: substance abuse } \\
\hline Evaluation & Instruments \\
\hline History-Level 0 & Screening for substance abuse \\
\hline \multirow[t]{3}{*}{ History-Level 1} & $\begin{array}{l}\text { Inventory: use and abuse of prescription medications, use of caffeine, nicotine, alcohol, and illicit } \\
\text { drugs }\end{array}$ \\
\hline & Adapted CAGE questionnaire for each substance $[50,51]$ \\
\hline & Stage of change for each substance, subjective \\
\hline \multirow[t]{2}{*}{ History—Level 2} & (i) Stage of change for each substance, objective \\
\hline & (ii) For smokers: Fagerström’s test of nicotine dependence $[52,53]$ \\
\hline \multicolumn{2}{|l|}{ History-Level 3} \\
\hline \multicolumn{2}{|l|}{ Examination-Level 0} \\
\hline \multicolumn{2}{|l|}{ Examination-Level 1} \\
\hline \multicolumn{2}{|l|}{ Examination-Level 2} \\
\hline \multirow[t]{3}{*}{ Examination-Level 3} & Nicotine-CO-oximetry \\
\hline & Alcohol—GGT, MCV \\
\hline & Drug levels in urine/blood/hair tests \\
\hline \multicolumn{2}{|c|}{ 7. Domain: other risk habits } \\
\hline Evaluation & Instruments \\
\hline \multicolumn{2}{|l|}{ History-Level 0} \\
\hline \multirow[t]{3}{*}{ History_Level 1} & (i) Medication adherence. \\
\hline & $\begin{array}{l}\text { (ii) Inventory of risk behaviors: risky sexual behavior, dangerous sports, gambling, dangerous driving, } \\
\text { potentially dangerous travel, internet/technology addiction. }\end{array}$ \\
\hline & (iii) Readiness to change-subjective \\
\hline History-Level 2 & Readiness to change-objective \\
\hline \multicolumn{2}{|l|}{ History-Level 3} \\
\hline \multicolumn{2}{|l|}{ Examination-Level 0} \\
\hline \multicolumn{2}{|l|}{ Examination-Level 1} \\
\hline \multicolumn{2}{|l|}{ Examination-Level 2} \\
\hline Examination-Level 3 & \\
\hline
\end{tabular}

[59], multiple behavioral assessment provides a composite index of overall behavior change and includes overarching outcome measures such as quality of life, related biometrics, and cost [60]. For example, a composite index for evaluating change in physical activity and diet showed that interventions focused only on exercise achieved a larger amount of behavior change than an intervention combining both physical activity and nutrition [60].

The eVITAL expert panel opted for a global impression rating of every major $\mathrm{HrH}$ and the graphical representation of the resulting lifestyle (health profile), instead of using composite indexes. Similar global ratings have been shown to be practical both in routine clinical practice [61] and in eHealth tools [62].

Unexpectedly given the income and the education level of the volunteers in our pilot sample, we found numerous $\mathrm{HrHs}$ in the "needs improvement" category, along with illnesses both related and unrelated to $\mathrm{HrH}$. We diagnosed one case each of prostate cancer and Sjögren's syndrome, as well as a high proportion of sleep, diet, and exercise problems. 
This pilot may indicate the relevance of designing both population- and primary care-based epidemiological studies of health lifestyles which include all basic habits and related conditions, as opposed to focusing specifically on targets such as nutrition or exercise.

4.1. Study Strengths. This study is unique in its integrative approach to the evaluation of $\mathrm{HrH}$ and the focus on the middle-aged adult population. It begins to address the barriers to health promotion in the primary care setting recently identified in Spain [63] by providing an innovative approach to the assessment of individuals. This preliminary taxonomy fills an existing gap in the assessment of $\mathrm{HrH}$. The eVITAL toolkit is freely available online for use in clinical and research settings, with the hope that this and other groups will continue to gather information on its utility and contribute to further refinement.

4.2. Study Limitations. The taxonomy has received adequate consensus, and the related tools included in EVITAL are those deemed by group to be most useful in the development of an integrated understanding of the $\mathrm{HrH}$ and lifestyle of an individual in the Spanish cultural context. However, the clinical utility of the toolkit as a whole will have to be validated in the future. The current, computerized version of eVITAL has not undergone the type of demonstration study reported here for the earlier assessment package prototype; this remains to be performed prior to widespread integration into clinical practice. The greatest limitation of the toolkit at this time is the concern raised about the feasibility of widespread use of eVITAL, most notably in populations with lower education level and socioeconomic status and in the primary care system. While it is important to gather enough information to develop a complete understanding of a patient's health lifestyle profile, the system we propose must be feasible within the existing health care system. eVITAL will continue to be adjusted to work toward this goal.

\section{Conclusion}

To our best knowledge this is the first toolkit of lifestyle and health-related habits based on a formal taxonomy of $\mathrm{HrH}$. This taxonomy may improve the assessment of lifestyle in health sciences, enhance the development of a classification of $\mathrm{HrH}$ and personal factors in the context of the WHO family of classifications, and develop this construct in new models of care such as person-centered medicine and diagnosis $[16,64]$.

\section{Abbreviations}

BMI: Body mass index

ICD: International Classification of Diseases

ICF: International Classification of Functioning, Disability and Health

HrH: Health-related habits

SPPI: Standardized Polyvalent Psychiatric Interview

WHO: World Health Organization.

\section{Conflict of Interests}

The authors declare that there are no conflicts of interest.

\section{Acknowledgments}

Funding for this study was provided by IMSERSO, the Spanish Ministry of Health and Social Policies and Equality. The participation of Caroly Walsh was funded by NIMH/NIH R25 MH071286 (Dr. Kerim Munir, PI). The funding body was not involved in study design, development of the eVITAL database, paper writing, or decision to submit for publication. The authors would like to acknowledge Dr. Kerim Munir and the members of the eVITAL group. Other members of the group are Jorge Moreno, Teresa Magallanes, Cristina Romero, Jaime Tarradellas, Juan Carlos Durán, Miriam Poole, Juan Carlos García-Gutiérrez, Félix Abad, Arun Mansukani, Francisco Cabello, Monserrat Manuvens, Eduard Estivill, Francisco Segarra, Javier Albares, Francisco Gil, María José Abellán, Saturnino Reyes, Carmen Vargas, Francisco Pradas, Carlos Mampel, Luisa Tejonero, and José Almenara Barrios.

\section{References}

[1] World Health Organization, The Global Burden of Disease, 2004 Update, Geneva, Switzerland, 2008.

[2] T. A. Pearson, S. N. Blair, S. R. Daniels et al., "AHA guidelines for primary prevention of cardiovascular disease and stroke: 2002 update: consensus panel guide to comprehensive risk reduction for adult patients without coronary or other atherosclerotic vascular diseases. American Heart Association Science Advisory and Coordinating Committee," Circulation, vol. 106, pp. 388-391, 2002.

[3] L. B. Goldstein, R. Adams, M. J. Alberts et al., "Primary prevention of ischemic stroke: a guideline from the American Heart Association/American Stroke Association Stroke Council: cosponsored by the Atherosclerotic Peripheral Vascular Disease Interdisciplinary Working Group; Cardiovascular Nursing Council; Clinical Cardiology Council; Nutrition, Physical Activity, and Metabolism Council; and the Quality of Care and Outcomes Research Interdisciplinary Working Group: the American Academy of Neurology affirms the value of this guideline," Stroke, vol. 37, pp. 1583-1633, 2006.

[4] M. J. Kelley and D. C. McCrory, "Prevention of lung cancer: summary of published evidence," Chest, vol. 123, no. 1, pp. 50S-59S, 2003.

[5] L. B. Yates, L. Djoussé, T. Kurth, J. E. Buring, and J. M. Gaziano, "Exceptional longevity in men: modifiable factors associated with survival and function to age 90 years," Archives of Internal Medicine, vol. 168, no. 3, pp. 284-290, 2008.

[6] M. J. Stampfer, F. B. Hu, J. E. Manson, E. B. Rimm, and W. C. Willett, "Primary prevention of coronary heart disease in women through diet and lifestyle," The New England Journal of Medicine, vol. 343, no. 1, pp. 16-22, 2000.

[7] J. J. Prochaska, C. R. Nigg, B. Spring, W. F. Velicer, and J. O. Prochaska, "The benefits and challenges of multiple health behavior change in research and in practice," Preventive Medicine, vol. 50, no. 1-2, pp. 26-29, 2010.

[8] L. Salvador-Carulla, A. Cano Sánchez, and J. Cabo-Soler, Longevidad: Tratado Integral Sobre Salud en la Segunda Mitad 
de la Vida, Editorial Médica Panamericana, Madrid, Spain, 2003.

[9] C. Bousquet, B. Trombert, A. Kumar, and J. M. Rodrigues, "Semantic categories and relations for modelling adverse drug reactions towards a categorial structure for pharmacovigilance," in Proceedings of the AMIA Annual Symposium, pp. 6165, 2008.

[10] S. Buetow, L. Kiata, T. Liew, T. Kenealy, S. Dovey, and G. Elwyn, "Patient error: a preliminary taxonomy," Annals of Family Medicine, vol. 7, no. 3, pp. 223-231, 2009.

[11] F. Alonso, C. O. Walsh, and L. Salvador-Carulla, "Methodology for the development of a taxonomy and toolkit to evaluate health-related habits and lifestyle (eVITAL)," BMC Research Notes, vol. 3, article 83, 2010.

[12] World Health Organization, International Classification of Functioning, Disability and Health (ICF), 2001.

[13] World Health Organization, Health Promotion Glossary, World Health Organization, Geneva, Switzerland, 1998.

[14] World Health Organization Noncommunicable Disease and Mental Health Cluster, Active Ageing: A Policy Framework, World Health Organization, 2002.

[15] World Health Organization, Promoting Mental Health: Concepts, Emerging Evidence, Practice, World Health Organization, Geneva, Switzerland, 2004.

[16] I. Salloum and J. Mezzich, "Person-centered diagnosis," International Journal of Integrated Care, vol. 10, pp. 75-78, 2010.

[17] J. O. Prochaska and W. F. Velicer, "The transtheoretical model of health behavior change," American Journal of Health Promotion, vol. 12, no. 1, pp. 38-48, 1997.

[18] J. O. Prochaska, S. Butterworth, C. A. Redding et al., "Initial efficacy of MI, TTM tailoring and HRI's with multiple behaviors for employee health promotion," Preventive Medicine, vol. 46, no. 3, pp. 226-231, 2008.

[19] T. Del Ser Quijano, F. Sánchez Sánchez, M. J. Garcia De Yébenes, Á. Otero Puime, M. V. Zunzunegui, and D. G. Muñoz, "Spanish version of the 7 Minute screening neurocognitive battery. Normative data of an elderly population sample over 70," Neurología, vol. 19, no. 7, pp. 344-358, 2004.

[20] P. R. Solomon, A. Hirschoff, B. Kelly et al., "A 7 minute neurocognitive screening battery highly sensitive to Alzheimer's disease," Archives of Neurology, vol. 55, no. 3, pp. 349-355, 1998.

[21] T. S. Altepeter, R. I. Adams, W. L. Buchanan, and P. Buck, "Luria memory words test and Wechsler memory scale: comparison of utility in dicriminating neurologically impaired from controls," Journal of Clinical Psychology, vol. 46, no. 2, pp. 190-193, 1990.

[22] A. L. Benton, The Revised Visual Retention Test, Psychological Corporation, New York, NY, USA, 1974.

[23] R. M. Reitan, "Validity of the Trail Making test as an indicator of organic brain damage," Perceptual \& Motor Skills, vol. 8, pp. 271-276, 1958.

[24] L. Dilks, J. Marceaux, B. Mayeaux, D. Turner, J. Bourassa, and M. Bourgeois, "Validity study of the western psychological services finger-tapping test," American Journal of Psychological Research, vol. 2, pp. 8-13, 2006.

[25] M. F. Scheier, C. S. Carver, and M. W. Bridges, "Distinguishing optimism from neuroticism (and trait anxiety, self-mastery, and self-esteem): a reevaluation of the life orientation test," Journal of Personality and Social Psychology, vol. 67, no. 6, pp. 1063-1078, 1994.

[26] J. Alonso, E. Regidor, G. Barrio, L. Prieto, C. Rodriguez, and L. De La Fuente, "Population-based reference values for the
Spanish version of the Health Survey SF-36," Medicina Clinica, vol. 111, no. 11, pp. 410-416, 1998.

[27] G. Vilagut, M. Ferrer, L. Rajmil et al., "The Spanish version of the Short Form 36 Health Survey: a decade of experience and new developments," Gaceta Sanitaria, vol. 19, no. 2, pp. 135-150, 2005.

[28] M. P. Beaudet, "Depression," Statistics Canada, vol. 7, no. 4, pp. 11-22, 1996.

[29] O. Dalgard, "Explanation of the Oslo-3 Social Support Scale (OSS-3),” In: System, E.U.P.H.I., (Ed.), 2006.

[30] A. S. Zigmond and R. P. Snaith, "The hospital anxiety and depression scale," Acta Psychiatrica Scandinavica, vol. 67, no. 6, pp. 361-370, 1983.

[31] P. T. Costa and R. R. McCrae, NEO PI-R Professional Manual, Psychological Assessment Resources, Odessa, Fla, USA, 1992.

[32] C. D. Ryff and C. L. M. Keyes, "The structure of psychological well-being revisited," Journal of Personality and Social Psychology, vol. 69, no. 4, pp. 719-727, 1995.

[33] D. van Dierendonck, D. Díaz, R. Rodríguez-Carvajal, A. Blanco, and B. Moreno-Jiménez, "Ryff's six-factor model of psychological well-being, a Spanish exploration," Social Indicators Research, vol. 87, no. 3, pp. 473-479, 2008.

[34] M. Hunter, “The Women's Health Questionnaire: a measure of mid-aged women's perceptions of their emotional and physical health," Psychology \& Health, vol. 7, pp. 45-54, 1992.

[35] J. P. Raynaud, J. Tichet, C. Born et al., "Aging male questionnaire in normal and complaining men," Journal of Sexual Medicine, vol. 5, no. 11, pp. 2703-2712, 2008.

[36] M. J. Herrero, J. Blanch, J. M. Peri, J. De Pablo, L. Pintor, and A. Bulbena, "A validation study of the hospital anxiety and depression scale (HADS) in a Spanish population," General Hospital Psychiatry, vol. 25, no. 4, pp. 277-283, 2003.

[37] T. H. Holmes and R. H. Rahe, "The social readjustment rating scale," Journal of Psychosomatic Research, vol. 11, pp. 213-218, 1967.

[38] C. Rodríguez-Sutil, P. Gil-Corbacho, and R. Martínez, "Presentación de la escala retiro de patrón de conducta TIPO A (ERCTA)," Psicothema, vol. 8, no. 1, pp. 207-213, 1996.

[39] M. Zuckerman, "Zuckerman-Kuhlman Personality Questionnaire (ZKPQ): an alternative five-factorial model," in Big Five Assessment, B. De Raad and M. Perugini, Eds., pp. 377-396, Hogrefe \& Huber, Seattle, Wash, USA, 2002.

[40] A. Lobo, R. Campos, M. J. Perez-Echeverria et al., "A new interview for the multiaxial assessment of psychiatric morbidity in medical settings," Psychological Medicine, vol. 23, no. 2, pp. 505-510, 1993.

[41] National Cancer Institute, "Breast cancer risk assessment tool: an interactive tool for measuring the risk of invasive breast cancer," In: Health, U.S.N.I.o., (Ed.).

[42] M. W. Johns, "A new method for measuring daytime sleepiness: the Epworth sleepiness scale," Sleep, vol. 14, no. 6, pp. 540-545, 1991.

[43] M. Ferrer, G. Vilagut, C. Monasterio, J. M. Montserrat, M. Mayos, and J. Alonso, "Measurement of the perceived impact of sleep problems: the Spanish version of the functional outcomes sleep questionnaire and the Epworth sleepiness scale," Medicina Clínica, vol. 113, no. 7, pp. 250-255, 1999.

[44] E. Chiner, J. M. Arriero, J. Signes-Costa, J. Marco, and I. Fuentes, "Validation of the Spanish version of the Epworth Sleepiness Scale in patients with sleep apnea syndrome," Archivos de Bronconeumología, vol. 35, no. 9, pp. 422-427, 1999. 
[45] Canadian Society for Exercise Physiology, "Physical Activity Readiness Questionnaire (PAR-Q),” 2002, http://www.csep .ca/.

[46] I. Trinidad Rodríguez, J. Fernández Ballart, G. Cucó Pastor, E. Biarnés Jordà, and V. Arija Val, "Validation of a short questionnaire on frequency of dietary intake: reproducibility and validity," Nutricion Hospitalaria, vol. 23, no. 3, pp. 242252, 2008.

[47] G. Bray, C. Bouchard, and W. P. T. James, Handbook Definitions and proponed current classifications of obesity, Marcel Dekker, New York, NY, USA, 1998.

[48] D. R. Matthews, METODO HOMA-IR, Homeostasis Model Assessment, 1985.

[49] B. E. Ainsworth, W. L. Haskell, M. C. Whitt et al., "Compendium of physical activities: an update of activity codes and MET intensities," Medicine and Science in Sports and Exercise, vol. 32, no. 9, pp. S498-S504, 2000.

[50] J. A. Ewing, "Detecting alcoholism. The CAGE questionnaire," Journal of the American Medical Association, vol. 252, no. 14, pp. 1905-1907, 1984.

[51] A. Rodríguez-Martos, R. Navarro, C. Vecino, and R. Pérez, "Validación de los cuestionarios KFA (CBA) y CAGE para diagnóstico del alcoholismo," Drogalcohol, vol. 11, pp. 132139,1986

[52] T. F. Heatherton, L. T. Kozlowski, R. C. Frecker, and K. O. Fagerstrom, "The Fagerstrom test for nicotine dependence: a revision of the Fagerstrom Tolerance Questionnaire," British Journal of Addiction, vol. 86, no. 9, pp. 1119-1127, 1991.

[53] E. Becoña and F. L. Vázquez, "The Fagerström Test for Nicotine Dependence in a Spanish sample," Psychological Reports, vol. 83, no. 3, pp. 1455-1458, 1998.

[54] G. Andrews, L. Perters, and M. Tesson, Measurement of Consumer Outcome in Mental Health: A Report to the National Mental Health Information Strategy Committee, Clinical Research Unit for Anxiety Disorders, Sydney, Australia, 1994.

[55] T. E. Seeman, B. H. Singer, C. D. Ryff, G. Dienberg Love, and L. Levy-Storms, "Social relationships, gender, and allostatic load across two age cohorts," Psychosomatic Medicine, vol. 64, no. 3, pp. 395-406, 2002.

[56] World Health Organization, International Classification of Diseases (ICD), World Health Organization, Geneva, Switzerland, 1994.

[57] J. Beddington, C. L. Cooper, J. Field et al., "The mental wealth of nations," Nature, vol. 455, no. 7216, pp. 1057-1060, 2008.

[58] L. Salvador-Carulla and V. Gasca, "Defining disability, functioning, autonomy and dependency in person-centered medicine and integrated care," International Journal of Integrated Care, vol. 10, pp. 69-72, 2010.

[59] C. Bridle, R. P. Riemsma, J. Pattenden et al., "Systematic review of the effectiveness of health behavior interventions based on the transtheoretical model," Psychology and Health, vol. 20, no. 3, pp. 283-301, 2005.

[60] J. J. Prochaska, W. F. Velicer, C. R. Nigg, and J. O. Prochaska, "Methods of quantifying change in multiple risk factor interventions," Preventive Medicine, vol. 46, no. 3, pp. 260-265, 2008.

[61] M. Berk, F. Ng, S. Dodd et al., "The validity of the CGI severity and improvement scales as measures of clinical effectiveness suitable for routine clinical use," Journal of Evaluation in Clinical Practice, vol. 14, no. 6, pp. 979-983, 2008.

[62] A. J. Rotondi, J. Sinkule, G. L. Haas et al., "Designing websites for persons with cognitive deficits: design and usability of a psychoeducational intervention for persons with severe mental illness," Psychological Services, vol. 4, no. 3, pp. 202224, 2007.

[63] G. Grandes, A. Sanchez, J. M. Cortada et al., "Is integration of healthy lifestyle promotion into primary care feasible? Discussion and consensus sessions between clinicians and researchers," BMC Health Services Research, vol. 8, article 213, 2008.

[64] J. E. Mezzich, I. M. Salloum, C. R. Cloninger et al., "Personcentred integrative diagnosis: conceptual bases and structural model," Canadian Journal of Psychiatry, vol. 55, no. 11, pp. 701-708, 2010. 


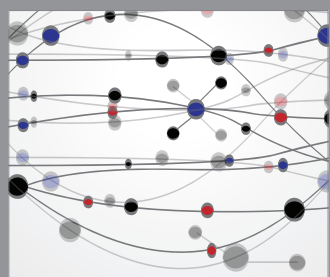

The Scientific World Journal
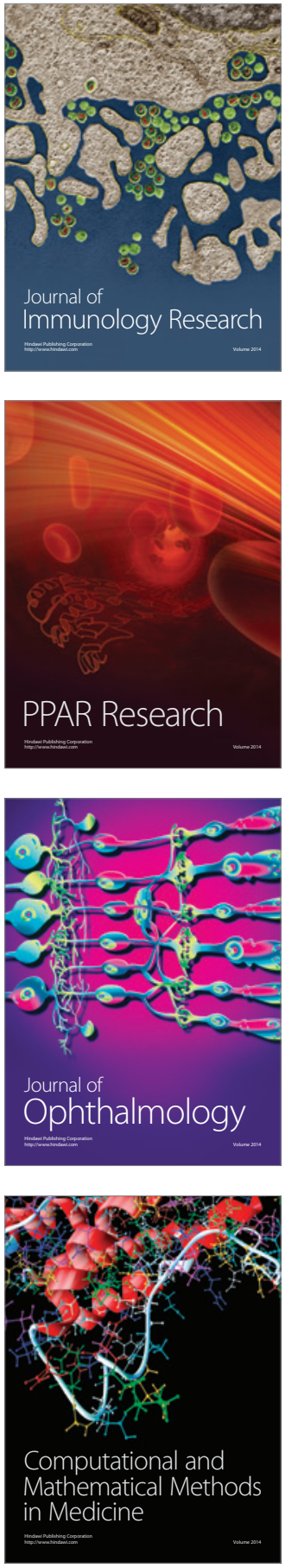

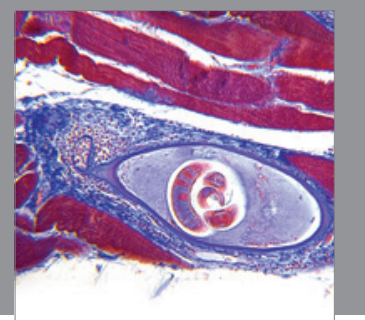

Gastroenterology

Research and Practice
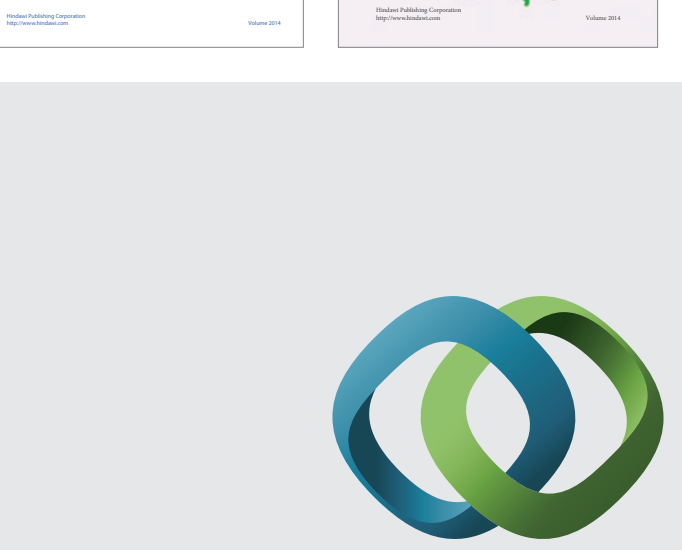

\section{Hindawi}

Submit your manuscripts at

http://www.hindawi.com
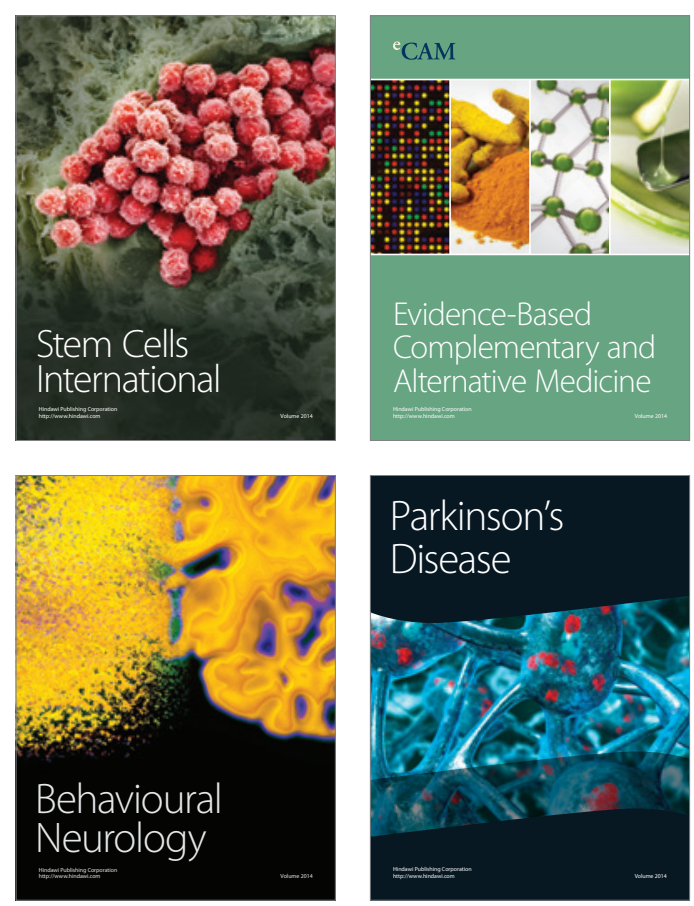

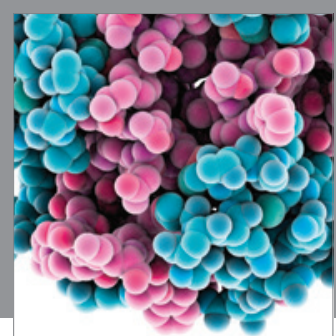

Journal of
Diabetes Research

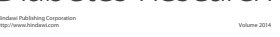

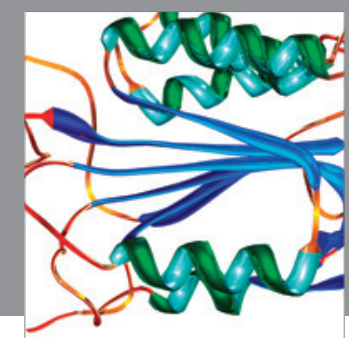

Disease Markers
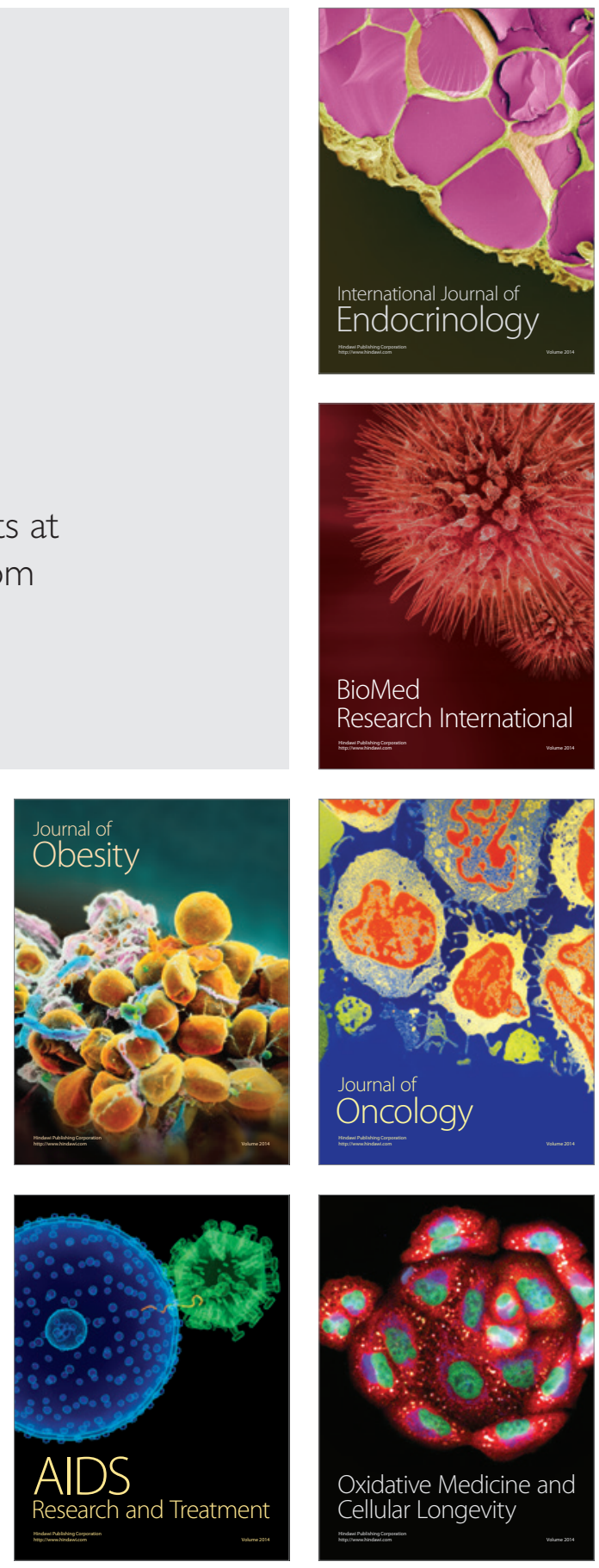